POS $\quad$ PROCEEDINGS

\title{
HAWC response to atmospheric electricity activity
}

\author{
Alejandro Lara* \\ Instituto de Geofísica, Universidad Nacional Autónoma de México \\ E-mail: alara@igeofisica.unam.mx
}

\section{Graciela Binimelis de Raga}

Centro de Ciencias de la Atmósfera, Universidad Nacional Autónoma de México

\section{Olivia Enríquez-Rivera}

Instituto de Geofísica, Universidad Nacional Autónoma de México

\section{for the HAWC collaboration}

The HAWC Gamma Ray observatory consists of 300 water Cherenkov detectors (WCD) instrumented with four photo multipliers tubes (PMT) per WCD. HAWC is located between two of the highest mountains in Mexico. The high altitude (4100 $\mathrm{m}$ asl), the relatively short distance to the Gulf of Mexico $(\sim 100 \mathrm{~km})$, the large detecting area $\left(22000 \mathrm{~m}^{2}\right)$ and its high sensitivity, make HAWC a good instrument to explore the acceleration of particles due to the electric fields existing inside storm clouds. In particular, the scaler system of HAWC records the output of each one of the 1200 PMTs as well as the 2, 3, and 4-fold multiplicities (logic AND in a time window of 30 ns) of each WCD with a sampling rate of $40 \mathrm{~Hz}$. Using the scaler data, we have identified 20 enhancements of the observed rate during periods when storm clouds were over HAWC but without cloud-earth discharges. These enhancements can be produced by electrons with energy of tens of $\mathrm{MeV}$, accelerated by the electric fields of tens of $\mathrm{kV} / \mathrm{m}$ measured at the site during the storm periods. In this work, we present the recorded data, the method of analysis and our preliminary conclusions on the electron acceleration by the electric fields inside the clouds.

35th International Cosmic Ray Conference - ICRC2017

10-20 July, 2017

Bexco, Busan, Korea

${ }^{*}$ Speaker. 


\section{Introduction}

Particle acceleration up to high energies inside the Earth's atmosphere has been observed by satellite gamma ray detectors during terrestrial gamma ray flashes [1, 2, 3, 4]. At ground level, high altitude cosmic ray detectors have reported ground enhancements during thunderstorms $[5,6,7,8]$.

The development of large electric fields during thunderstorms (up to $200 \mathrm{kV} / \mathrm{m}$ [9]) accelerates charged particles. Electrons may gain energies up to tens of $\mathrm{MeV}$ [7]. In this work we report enhancements of the count rates observed by the High Altitude Water Cherenkov Observatory (HAWC) which might be related to the atmospheric electric field.

HAWC is an air shower detector located at 4,100 m a.s.l, N $18^{\circ} 59^{\prime} 48^{\prime \prime}$, W $97^{\circ} 18^{\prime} 34^{\prime \prime}$. Built on the slope of Sierra Negra, Puebla in Mexico it consists of 300 water Cherenkov detectors $7.3 \mathrm{~m}$ diameter and $4.5 \mathrm{~m}$ deep. Each tank is filled with filtered water and the total detector comprises an extension of $22,000 \mathrm{~m}^{2}$.

HAWC is operating on one of the highest mountains in Mexico. HAWC's high altitude together with the proximity of the Gulf of Mexico make the array an excellent laboratory to study the high energetic processes during thunderstorms. In section 2 we discuss the weather on the HAWC site in more detail.

In section 3 we describe HAWC scaler systems which are able to detect low energy particles. In Sec. 4 we present the enhancements of the scaler count rates observed by HAWC due to the presence of strong electric fields. Finally our discussion is presented in Sec. 5

\section{The weather at the HAWC site}

Southern and Central Mexico is located in the tropics, with ample humidity during most of the year and characterized by a 6-month rainy season. HAWC in particular, is located in a region with frequent presence of clouds formed by forced orographic lifting or due to atmospheric convective instability. The latter mechanism often leads to cumulus clouds responsible for the development of precipitation and are also responsible for charge separation within the cloud, due to collisions between hydrometeors at the different temperature ranges observed. The development of poles of positive and negative charge within the cloud give rise to an electric field that can reach breakdown point and result in a lightning discharge, within the cloud or from the cloud to ground.

Several ground-based networks have been developed to monitor cloud-to-ground lightning continuously to assess the risk to the population. One such global network is the World Wide Lightning Location Network (WWLN), documented by Dowden et al (2008). The first studies of combined precipitation and lightning over Mexico were carried out in 2010 and revealed the regions of the country where most of the cloud-to-ground lightning is observed (Kucienska et al, 2010). Furthermore, Raga et al (2014) showed that Mexico is particularly vulnerable, with a large number of deaths per year.

While HAWC is not located in the region of highest incidence of lightning in Mexico, its location on the Sierra Negra ensures that it will be affected by electrically-charged clouds and lightning for about 6 months of the year. The electric fields associated with the convective clouds and the proximity with intra-cloud and cloud-to-ground lightning provide a unique setting to study the effect of these phenomena on the measurements made by HAWC. In this work, we carried 
out measurements of the ambient variables using a weather station placed at the HAWC site. To measure the electric field, we used an electric field mill Boltek-100 installed at the eastern side of the array. Plots of these variables are shown in section 4.

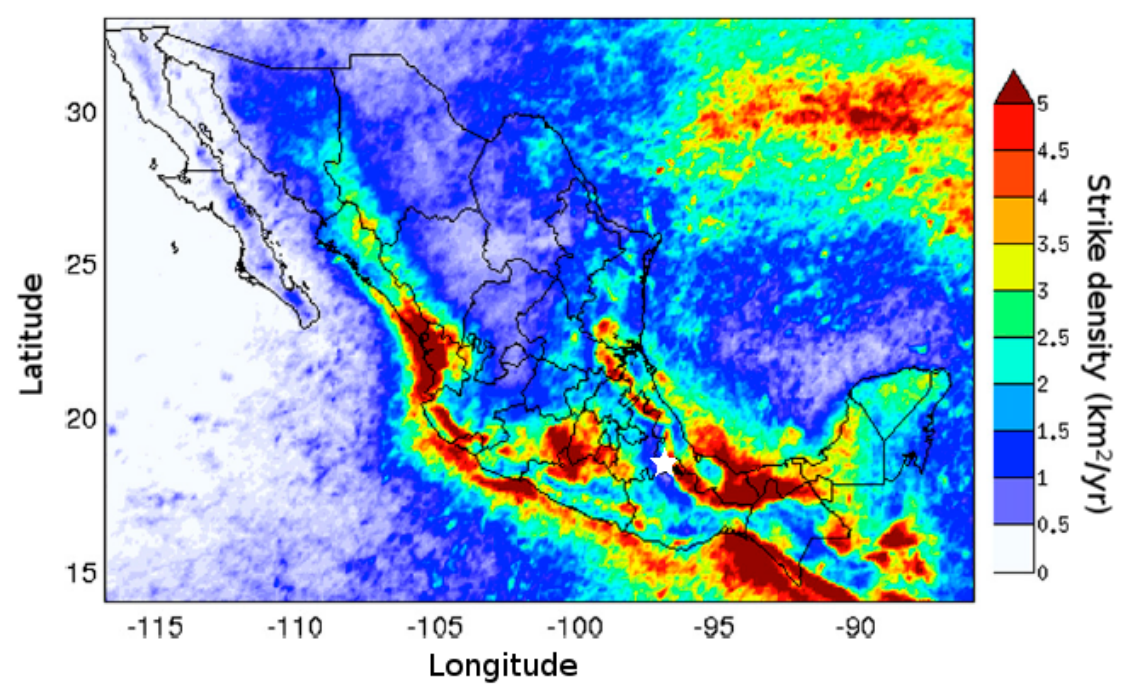

Figure 1: Average spatial distribution of cloud-to-ground lightning density in flashes per square kilometer per year for the period 2006-2012, adapted from Raga et al (2014). The white star mark the location of HAWC.

\section{HAWC scaler systems}

HAWC data are collected by two data acquisition systems (DAQs). The main DAQ measures arrival times and time over thresholds of PMT pulses and allows for the reconstruction of the air shower arrival direction and energy of the primary particle. The electronics are based on time to digital converters (TDC). The main DAQ also has a TDC scaler system which counts the hits inside a time window of $30 \mathrm{~ns}$ of each PMT and the coincidences of 2, 3 and 4 PMTs in each water Cherenkov detector. These coincidences are called multiplicity 2, 3 and 4, respectively. The secondary DAQ consists of a counting system that registers each time the PMT is hit by $>1 / 4$ photoelectron charge and we call it hardware (HW) scaler system. This simpler system together with the TDC scalers allows one to measure particles below the energies of reconstructable showers.

\section{Count rate enhancements}

We have noted that the HAWC scaler systems responds to the atmospheric electricity at least in four ways: i) when the electric field is positive or weak negative the count rate does not suffer any change as seen in Figure 2 where we have plotted the TDC scaler rates during the negative electric field enhancement observed on Nov 22, 2014. All scaler rates are in percentage taking as reference, i.e. $100 \%$, the mean scaler rate calculated one hour before the event started. The electric field is shown in black solid line. As an eye aid, the equivalent zero electric field is plotted 


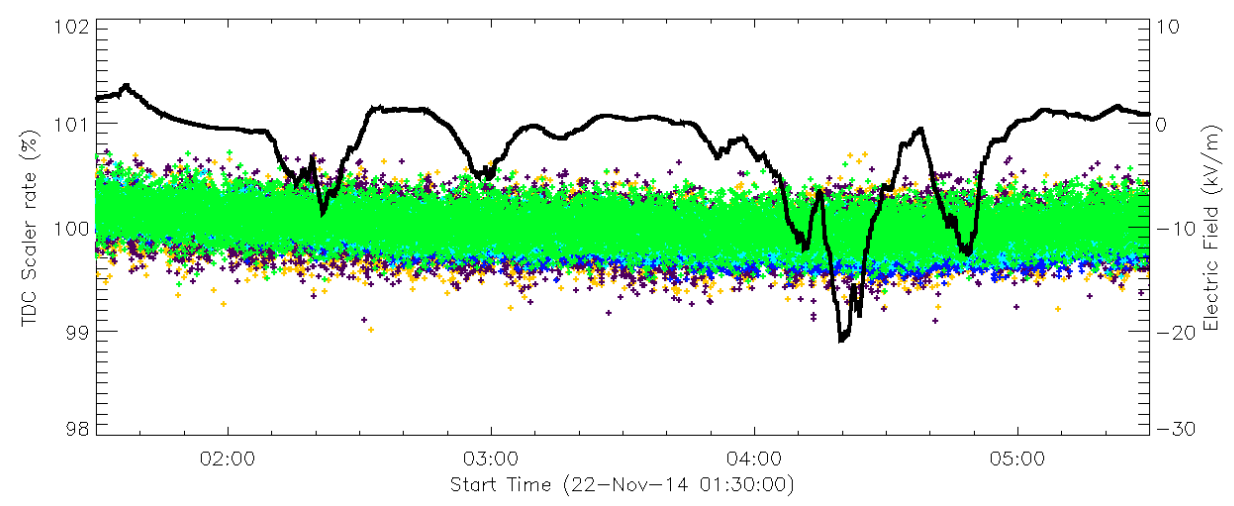

Figure 2: Mean count rate of the TDC scaler Multiplicities: 2 (blue), 3 (cyan) and 4 (green); and rates of the 8" (orange) and 10" (purple) PMTs, during November 22, 2014 when a moderate negative electric field was observed (black curve).

as a horizontal dashed line. ii) when the storm is very close to the array with large amount of discharges, the system behavior is unstable and the system restarts frequently. There are scaler enhancements but they may be due to the discharges and/or the electric field or electromagnetic noise. For example, Figure 3 shows the TDC scaler rates during November 8, 2014, an active day in terms of atmospheric electricity. A thunderstorm took place during this period as seen by the red square symbols and purple triangles representing the cloud to ground and inter-cloud discharges, respectively. There were nearby discharges as shown by the rapid variations of the field strength (it is important to note that the electric field detector gets saturated when the electric field is larger than $\pm 40 \mathrm{kV} / \mathrm{m})$.

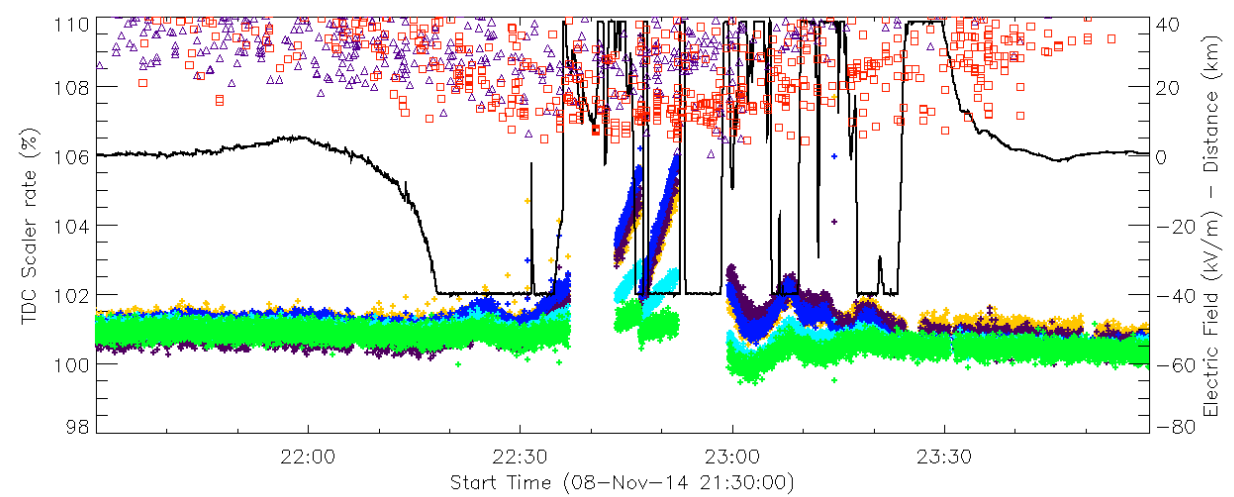

Figure 3: TDC scaler rates and electric field measured during Nov 8, 2014. The color code is similar as Fig. 2. The distance of the reported cloud to ground (red squares) and inter-cloud (purple triangles) discharges are also plotted.

There are some events where the atmospheric electric activity is not so strong and therefore, the response of the scaler system is somehow "well behaved." In those cases we can distinguish: iii) a fast response of the scaler system associated to the discharges and iv) a slow response to strong negative electric fields. These responses are depicted in Figure 4 where we have plotted, 
with colored dots, the count rate (in percentage) of each channel of the HW scaler system during September 18, 2015. The electric field shows two rapid changes around 00:45, associated with discharges. The squares and triangles indicate that a storm took place. There are sharp scaler enhancements associated with the closest discharges between 00:35 and 00:50 UT.

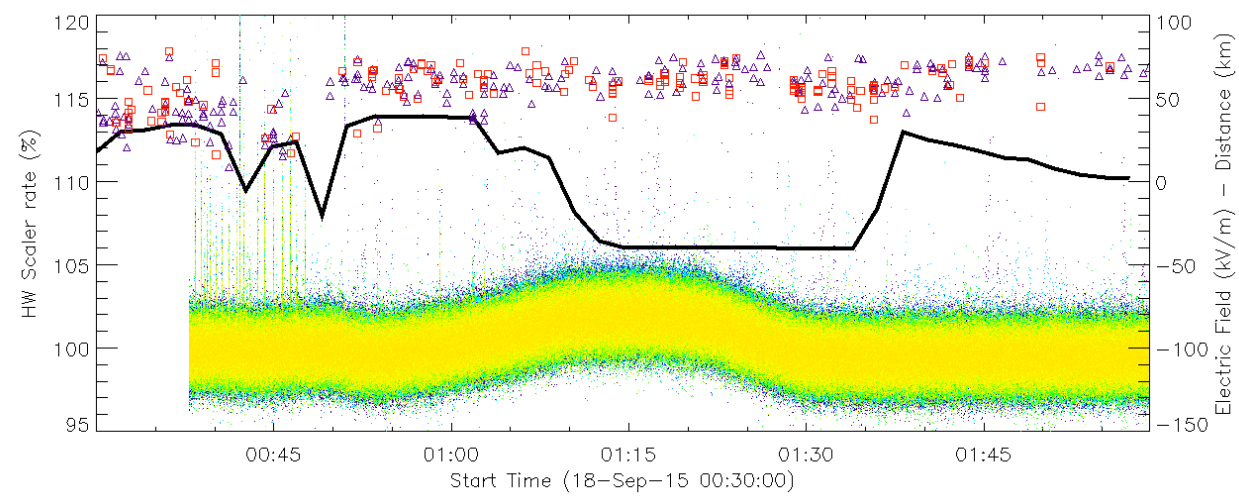

Figure 4: Mean count rate of the HW scaler system, each available channel is plotted in colored dots. The distance of the reported cloud to ground (red squares) and inter-cloud (purple triangles) discharges are also plotted.

In this work we focus on the slow response of the scaler system due to the presence of strong negative electric field. A clear example of this slow response was observed during May 26, 2015 and is depicted in Figures 5 and 6 for TDC and HW scalers, respectively. We have selected this event due to the fact that there is no saturation of the electric field measurements.

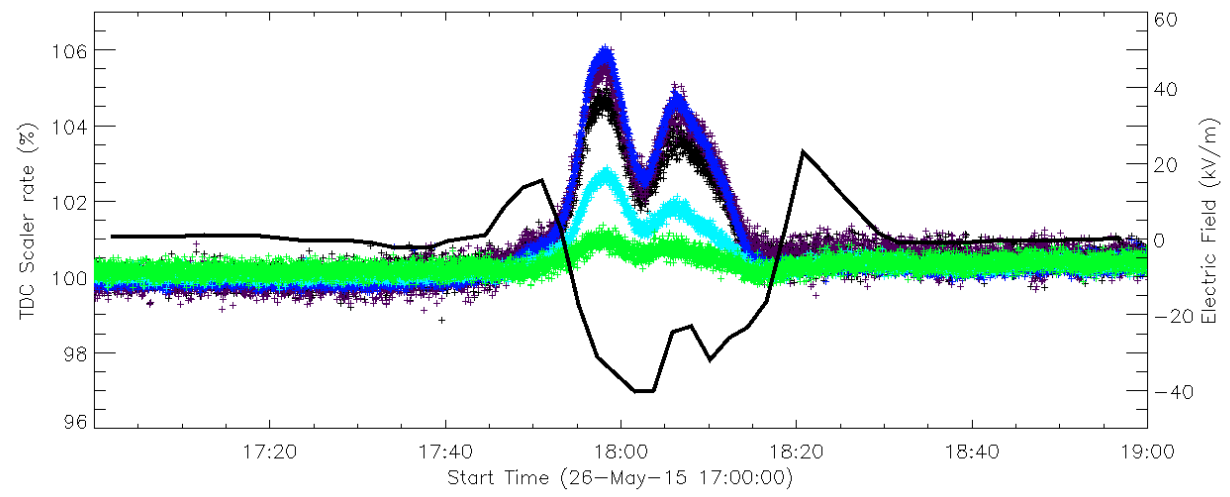

Figure 5: Mean count rate of the TDC scaler Multiplicities during May 26, 2015. The color code is the same as in Figure 2.

The environment parameters during May 26, 2015 are presented in Figure 7. From top to bottom, we plotted the electric field, pressure, temperature, humidity, rain fall and solar irradiance. The latter two are displayed in order to show the presence of clouds at the site during the scaler enhancements. In particular, it is well known that the count rate of cosmic ray detectors has an inverse dependence on the ambient pressure. This figure shows that the count rate enhancements during the events are not related to pressure changes. 


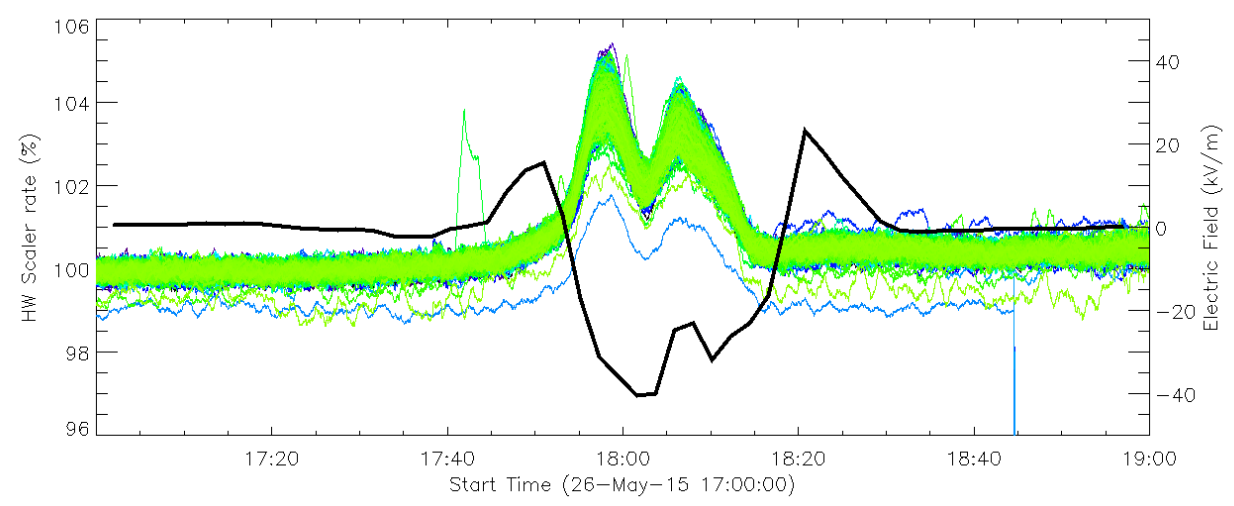

Figure 6: Mean count rate of the HW scaler system during May 26, 2015. The color code is the same as in Figure 4.
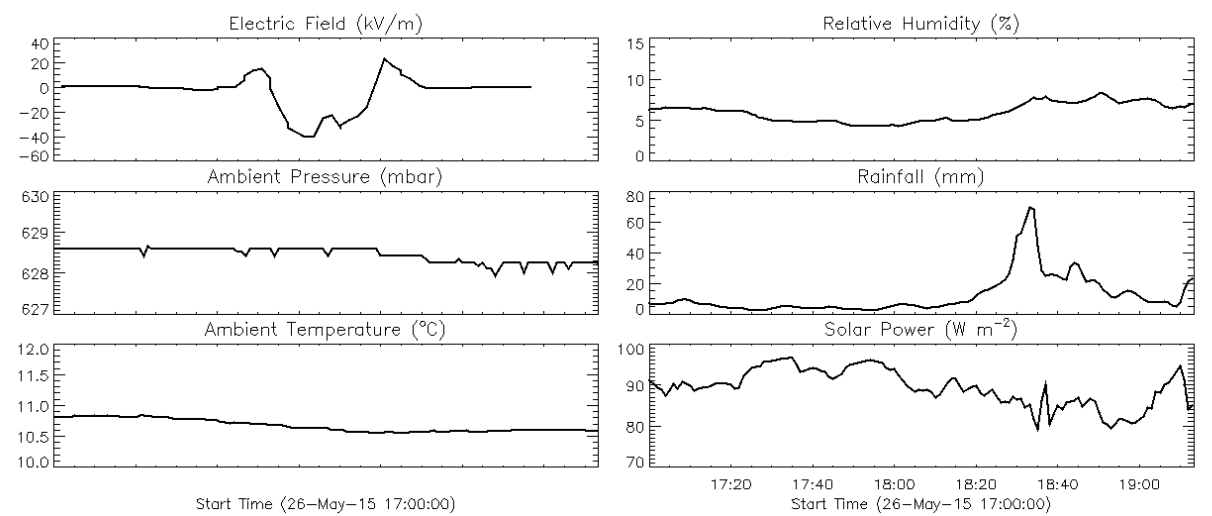

Figure 7: Environment variables at the HAWC site during May 26, 2015. From top to bottom are: Electric field, ambient pressure, temperature (left panels), humidity, rainfall and solar irradiance (right panels).

Figure 8 shows the scatter plot of the TDC scaler enhancement as a function of the strength of the electric field, during the May 26 event. Unfortunately the electric field data are stored with poor time resolution ( $\sim 1.5$ minutes), limiting the statistics available for correlations. In order to show the tendency of the correlation, we fit a second degree polynomial to each mean multiplicity and mean PMT rates. The correlation coefficients are shown in the plot as reference. The scatter is high but one can see that both 10" PMT and multiplicity 2 rates are more affected by the electric field enhancement. The 8" PMTs, multiplicity 3 and finally multiplicity 4 are less affected. If the rate enhancements are being produced by the acceleration of charged particles in the electric field, this correlation would indicate more low-energy and few high-energy particles in the enhancement. Finally, the small scatter plots in Figure 8 emphasize the lack of correlation between the scaler rates and the Pressure/Temperature measured at the site.

\section{Discussion}

In this work, we presented examples of the HAWC scaler system response to the atmospheric 


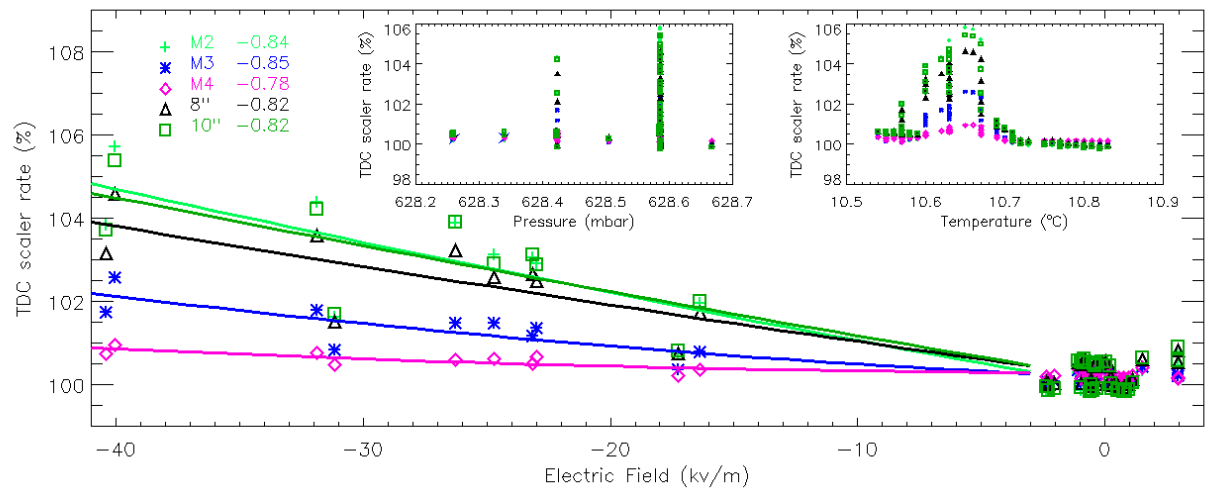

Figure 8: Scatter plot of the TDC scale Multiplicity 2 (green), 3 (blue) 4 (magenta); and 8" (black) and 10" PMT rates as a function of the electric field during May 26, 2015. The correlation coefficients are shown next to each Multiplicity and PMT set. Upper panels: scatter plots of the scaler rates vs Pressure (left) and Temperature (right)

electricity activity. We showed an example of the rapid response of the HW scaler system due to a close lightning activity (Figure 4). However, in this work we focus in the scaler system response to the negative electric field.

In particular we presented an example in which the electric field was not saturated and was observed by the two scaler systems. The correlated enhancement of all the available PMTs of the array seen by the HW scaler system (Figs. 6) shows that the enhancement embraces the entire array with similar response at time scales of seconds. The preliminary correlation analysis between the negative electric field and TDC count rates shows the high relationship between these variables (Figures 8). Furthermore, the absence of correlation between the scaler count rates and atmospheric variables such as pressure or temperature supports a possible scenario where the scaler rate enhancements might be produced by particle acceleration due to the electric field of clouds observed by HAWC. If our hypothesis is correct, the enhancements of all multiplicities of the TDC scaler system (Fig. 5) will allow us to determine the energy of the incident particles.

We have shown that HAWC can be a good instrument to study the acceleration of particles by the atmospheric electricity. It is necessary to perform a detailed analysis and simulations to quantify our observations, as well as rule out instrumental effects in the photomultipliers (such as inductive charging) that could be producing the observed rate enhancements. This analysis will be published elsewhere.

\section{Acknowledgments}

We acknowledge the support from: the US National Science Foundation (NSF); the US Department of Energy Office of High-Energy Physics; the Laboratory Directed Research and Development (LDRD) program of Los Alamos National Laboratory; Consejo Nacional de Ciencia y Tecnología (CONACyT), México (grants 271051, 232656, 260378, 179588, 239762, 254964, 271737, 258865, 243290, 132197), Laboratorio Nacional HAWC de rayos gamma; L'OREAL Fellowship for Women in Science 2014; Red HAWC, México; DGAPA-UNAM (grants IG100317, IN111315, IN111716-3, IA102715, 109916, IA102917); VIEP-BUAP; PIFI 2012, 2013, PROFICIE 2014, 2015; the University of Wisconsin Alumni Research Foundation; the Institute of 
Geophysics, Planetary Physics, and Signatures at Los Alamos National Laboratory; Polish Science Centre grant DEC-2014/13/B/ST9/945; Coordinación de la InvestigaciÃşn Científica de la Universidad Michoacana. Thanks to Luciano Díaz and Eduardo Murrieta for technical support.

\section{References}

[1] G. Fishman, P. Bhat, R. Mallozzi, J. Horack, T. Koshut, C. Kouveliotou, G. Pendleton, C. Meegan, R. Wilson, W. Paciesas, et al., Science 264, 1313 (1994).

[2] D. M. Smith, L. I. Lopez, R. P. Lin, and C. P. Barrington-Leigh, Science 307, 1085 (2005)

[3] M. Marisaldi, F. Fuschino, C. Labanti, M. Galli, F. Longo, E. Del Monte, G. Barbiellini, M. Tavani, A. Giuliani, E. Moretti, et al., J. Geophys. Res. 115, A00E13 (2010)

[4] M. S. Briggs, G. J. Fishman, V. Connaughton, P. N. Bhat, W. S. Paciesas, R. D. Preece, C. Wilson-Hodge, V. L. Chaplin, R. M. Kippen, A. von Kienlin, et al., J. Geophys. Res. 115, A07323 (2010) [11]

[5] V. V. Alexeenko, N.S. Khaerdinov, A.S. Lidvansky and V.B.Petkov, Transient variations of secondary cosmic rays due to atmospheric electric field and evidence for pre-lightning particle acceleration, Phys. Lett. A, 301 (2002) [12]

[6] S. Vernetto for EAS-TOP Collaboration, The EAS counting rate during thunderstorms. Proc. of 27th ICRC,Copernicus Gesellschaft, Hamburg, Germany,August 7-15, 10 (2001) [17]

[7] A. Chilingarian, B. Mailyan and L. Vanyan, Recovering of the energy spectra of electrons and gamma rays coming from the thunderclouds, Atmos. Res., 114-115 (2012) [18]

[8] H. Tsuchiya, K. Hibino and K. Kawata et al., Observation of thundercloud-related gamma rays and neutrons in Tibet, Phys. Rev. D, 85 (2012)

[9] M. Stolzenburg, T. C. Marshall, W. D. Rust, E. Bruning, D. R. MacGorman and T. Hamlin, Electric field values observed near lightning flash initiations, Geophys. Res. Lett. 34 (2007) L04804.

[10] Boccippio, Dennis, J., Kenneth L. Cummins, Hugh J. Christian, and Steven J. Goodman, 2001: Combined Satellite- and Surface-Based Estimation of the IntracloudâĂŞCloud-to-Ground Lightning Ratio over the Continental United States. Mon. Wea. Rev., 129, 108 âĂŞ122.

[11] Christian, H. J., Blakeslee R. J., Boccippio D. J., Boeck W. L., Buechler D. E., Driscoll K. T., Goodman S. J., Hall J. M., Koshak W. J., Mach D. M., and Stewart M. F., 2003, Global frequency and distribution of lightning as observed from space by the Optical Transient Detector. J. Geophys. Res., 108(D1), 4005.

[12] Dowden, R. and 30 more authors, 2008: World-Wide Lightning Location using VLF propagation in the Earth-Ionosphere waveguide. IEEE Antennas and Propagation Mag., 50, 40-60.

[13] Kucienska, B., G. B. Raga, and O. Rodriguez, 2010: Cloud-to-ground lightning over Mexico and adjacent oceanic regions: a preliminary climatology using the WWLLN dataset. Ann. Geophys., 28, $2047 \hat{a ̂ A S} 2057$.

[14] Raga, G.B., M.G. de la Parra and B. Kucienska, 2014: Deaths by lightning in Mexico (1979-2011): Threat or vulnerability? Wea. Climate Soc, 6, 434-444. 